\title{
First Responder - ein erweitertes Konzept in der Notfall-Hilfe
}

\author{
Hugo Saner ${ }^{a}$, Beat Baumgartner \\ a Prof. Dr. med., Universitätsklinik für Kardiologie, Inselspital Bern, Projektleiter Herznotfall Herzstiftung Olten \\ ${ }^{b}$ Rettungsdienst Spital STS AG, Gesigen, Präsident First Responder Kanton Bern
}

Vor über 20 Jahren gab es in der Schweiz erste Initiativen, um ein flächendeckendes System von medizinischen Laien als Ersthelfer (First Responder, FR) für Herznotfälle ausserhalb des Spitals aufzubauen. Dies mit dem Ziel, die Überlebensrate bei Herzstillständen mittels früher CPR (Cardiopulmonary Resuscitation) und früher Defibrillation am Ereignisort zu steigern und das Risiko von Hirnschädigungen zu reduzieren. Die positive Bilanz aus den Einsätzen der Ersthelfer lässt die Frage nach einer Erweiterung ihres Einsatzgebietes aufkommen.

Ab dem Jahr 2000 wurden Angehörige der Feuerwehren der Region Olten als FR bei Herznotfällen ausgebildet und als Herznotfallgruppen in das Alarmdispositiv integriert. Eine Untersuchung über die Jahre 20012008 zeigte eine deutliche Steigerung der Überlebensrate beim Herzstillstand ausserhalb des Spitals [1]. Der nächste Entwicklungsschritt erfolgte im Kanton Tessin, wo flächendeckend öffentlich zugängliche Defibrillatoren installiert und Laien als FR in Ergänzung eingesetzt werden [2]. Die Überlebensrate konnte so von 15 auf 55\% gesteigert werden. Entscheidend ist aber auch hier der Einsatz von FR aus den Blaulicht-Organisationen Polizei, Feuerwehr und Grenzwacht, die bei über 80\% der erfolgreichen Einsätze beteiligt sind.

2014 publizierte der Interverband für Rettungswesen erstmals einen Leitfaden für FR-Systeme in der Schweiz mit Neuauflage 2018 [3]. Die Alarmierung der FR erfolgt durch die Alarmzentralen (AZ) 144 zeitgleich mit der Alarmierung des Rettungsdienstes. Mittels einer App bestätigt der FR seine Einsatzbereitschaft und erhält die nötigen Einsatzinformationen. Derzeit bestehen in 17 Kantonen FR-Netzwerke oder sind im Aufbau [4]. Im Kanton Bern stehen über 2000 FR im Einsatz (www.firstresponder.be).
Bis vor kurzem beschränkte sich der Begriff FR auf ErstHelfer für Einsätze bei Verdacht auf Herz-KreislaufStillstand oder Thoraxschmerz. Das Einsatzpotential für FR geht aber über die Herznotfälle hinaus.

\section{Einsatzgebiete von First Respondern erweitern}

Bei den Laien-FR handelt es sich um Freiwillige. Durch ihre Bereitschaft, oft rund um die Uhr zur Verfügung zu stehen, nehmen sie bewusst eine Einschränkung ihrer persönlichen Freiheit und möglicherweise belastende Einsatzerfahrungen in Kauf. Dieses menschliche Potential kann in unserer Gesellschaft über den Herznotfall hinaus überall dort, wo rasche Hilfe notwendig werden kann, genutzt werden. Die FR sind immer als Ergänzung und nie als Ersatz zu bestehenden Systemen zu betrachten.

\section{Ersthelfer bei häuslichen Notfällen}

Alleinstehende ältere Menschen, aber auch andere hilfsbedürftige Personen besitzen oft einen Alarmknopf, über den im Notfall ein Callcenter alarmiert

Zusätzliche Einsatzmöglichkeiten der First Responder

\begin{tabular}{lll}
\hline Herznotfälle (bisher) & Häusliche Notfälle & Grossereignisse/Katastrophen \\
Einsatzindikation: & Einsatzindikation: Alarmknopf ausgelöst, & Einsatzindikation: Betreuung von leicht- oder \\
Brustschmerz, Bewusst- & keine Kontaktperson zeitnah verfügbar & unverletzten Personen, wenn die personellen \\
losigkeit, Atemnot, & & Ressourcen der offiziellen medizinischen \\
Herznotfall & & Notfallorganisation ausgeschöpft sind \\
\hline
\end{tabular}


werden kann. Neuere Entwicklungen nutzen nicht-invasive Sensorsysteme, die auch Notrufe auslösen können, wenn der Betroffene nicht in der Lage ist, den Notfallknopf zu drücken. Das Callcenter nimmt mit dem Betroffenen Kontakt auf und informiert Angehörige, Nachbarn oder weitere Kontaktpersonen. Wenn diese nicht oder nicht innert nützlicher Frist aufgeboten werden können und dadurch die Hilfeleistung verzögert wird, kann der Einsatz von FR eine wertvolle Ergänzung sein. Dabei kontaktiert das Callcenter die für die Region zuständige Alarmzentrale zur Alarmierung der FR. Ein erster Pilotversuch wurde im Dezember 2019 im Kanton Bern gestartet und ist auch im Kanton Solothurn in Vorbereitung.

Die Einsätze der FR bei häuslichen Notrufen beruhen wie bei Herznotfällen auf den Grundsätzen der Freiwilligkeit und Menschlichkeit. Es geht bei beiden Einsatzformen um Hilfeleistungen, bei denen kein professionelles medizinisches Wissen vorausgesetzt wird. Bei der Alarmierung ist der Zeitfaktor für die Hilfeleistung von zentraler Bedeutung.

Folgende Voraussetzungen wurden im Kanton Bern für die Erweiterung der Einsatzindikation definiert und sind auch für weitere Regionen von Bedeutung:

- Ergänzung der Ausbildungsangebote für FR: Es muss eine praxisnahe Weiterbildung zum Thema Notruf bei häuslichen Alarmen angeboten werden (z.B. Hilfe für eine gestürzte Person, Umgang mit verwirrten Personen, Indikationen für die Alarmierung des Rettungsdienstes usw.).

- Anpassung des Alarms: Der Alarm erfolgt via Empfänger des Notrufes (z.B. Rotkreuz-Notruf, Medicall, weitere Callcenter) und wird bei Bedarf an die Alarmzentrale zur Auslösung des Aufgebotes weitergeleitet. Damit beim Alarm über die Notfall-App die Indikation Herz-Kreislauf-Stillstand/Thoraxschmerz vom häuslichen Notrufalarm unterschieden werden kann, werden auf der App der FR verschiedene Alarmtöne definiert. Der Algorithmus für den Alarmierungsprozess muss entsprechend angepasst werden.

- Sichern der Finanzierung: Während die Einsätze der FR auch bei dieser Indikation freiwillig und unentgeltlich sind, fallen Kosten für die Anpassung der App (in der Regel momentum) und der Einsatzsysteme sowie die Info und Schulung aller Beteiligten an.

\section{Grossereignisse und Katastrophen}

Für Grossereignisse werden in der Schweiz seit längerem spezielle Notfallkonzepte entwickelt, welche auch die sanitätsdienstliche Versorgung im Katastrophenfall sicherstellen sollen. Auch auf grössere Schadensereignisse mit Toten und Verletzten ausserhalb der Agglomerationen sind die kantonalen Katastrophen- und Rettungsdienste in der Regel gut vorberei-

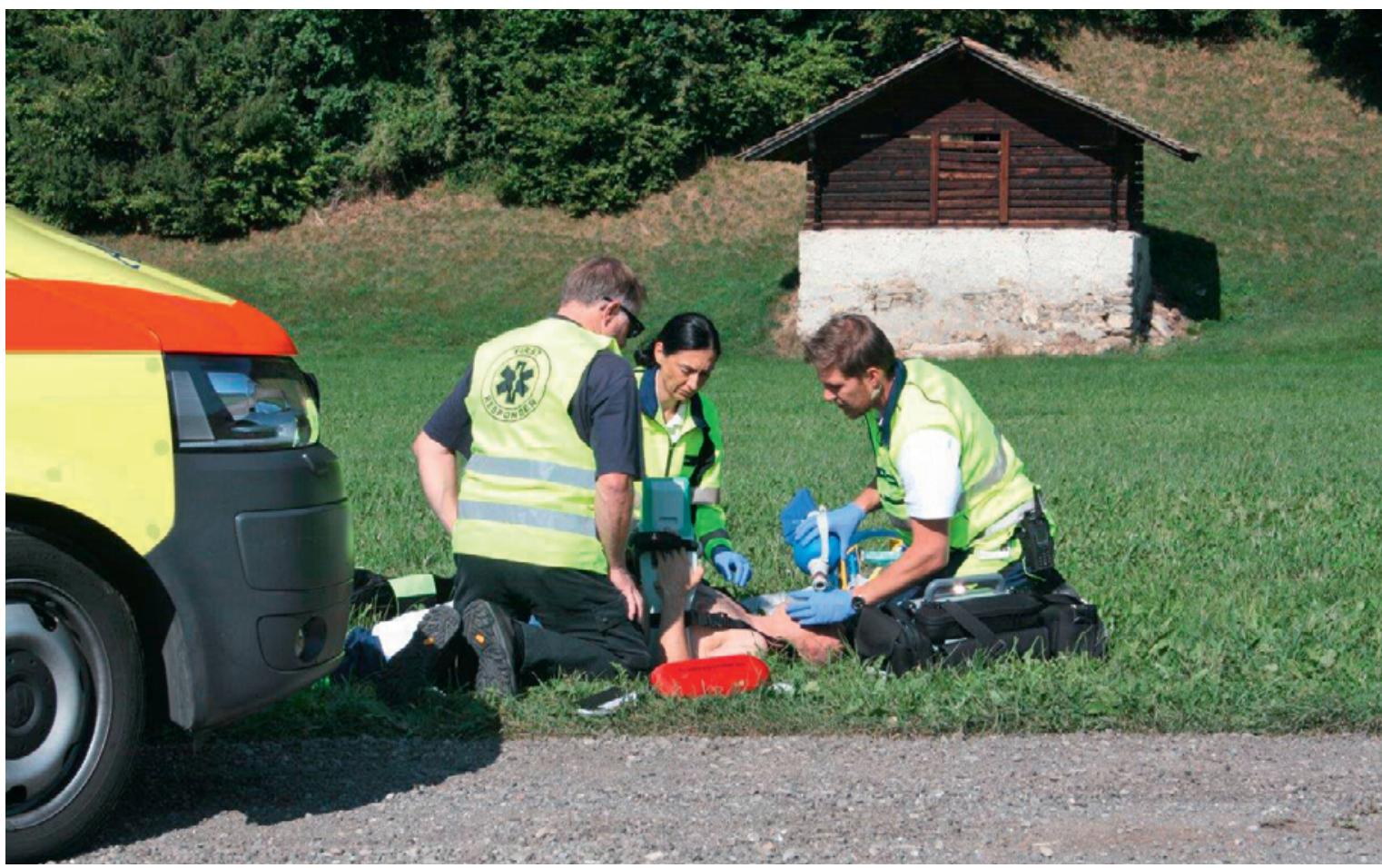

Schnelle Hilfe, grosse Wirkung: Gerade an abgelegenen Orten sind First Responder eine wertvolle Ergänzung zu den offiziellen Rettungsdiensten. 
tet. Dies schliesst aber nicht aus, dass es trotz optimaler Planung zu Situationen kommen kann, bei denen ein zeitgerechter Einsatz der professionellen Rettungsdienste nicht gesichert ist. Dies kann viele Ursachen wie z.B. Probleme und Behinderungen bei den Anfahrtswegen haben. Wenn sich in solchen Situationen FR in der Nähe des Ereignisortes aufhalten, können sie wertvolle erste Notfallhilfe leisten.

Auch für diese Indikation müssen ein spezielles Alarmkonzept erarbeitet und eine zusätzliche von den übrigen Alarmtönen unterscheidbare Alarmierung auf der Notfall-App installiert werden.

Die Einsätze der FR bei Grossereignissen und Katastrophen kann dort sinnvoll sein, wo erweiterte personelle Ressourcen benötigt werden. In etlichen Regionen des Berner Oberlands bestehen seit vielen Jahren sogenannte "Einsatzgruppen S+», welche von aktiven Samaritern gebildet werden. Die Mitglieder unterstützen den Aufbau, Betrieb und Abbau einer Sanitätshilfestelle - sie betreuen leichtverletzte Personen und unterstützen die professionellen Rettungsdienstmitatbeitenden bei der Patientenversorgung. Bei ausgelöstem Alarm ist der Zeitfaktor für die Hilfeleistung von zentraler Bedeutung. Auch für diese Einsatzindikation ist eine spezielle Ausbildung für die FR mit den entsprechenden Schwerpunkten zu planen. Wiederholtes Üben mit professionellen Helfern ist in diesem Bereich zwingend.

\section{Menschliches Potential nutzen}

Auch in der Schweiz stellen sich immer mehr medizinische Laien als FR zur Verfügung. Sie sind bereit, sich in Notfällen alarmieren zu lassen und falls nötig freiwillig und unentgeltlich Ersthilfe-Einsätze zu leisten. Dieses menschliche Potential kann in unserer Gesell- schaft über den Herznotfall hinaus genutzt werden. Dies überall dort, wo rasche Nachbarschaftshilfe in Notfällen nötig ist und Ergänzungen zum öffentlichen Rettungswesen sinnvoll sind. Ein zusätzlicher Bedarf nach Ersthelfern besteht bei häuslichen Notfällen, wenn keine Nachbarn, Angehörigen oder weitere Hilfspersonen zur Verfügung stehen oder diese nicht zeitgerecht aufgeboten werden können. Diese Einsätze können entscheidend zur Verbesserung der Sicherheit von alleinstehenden, meist älteren Personen in unserer Gesellschaft beitragen. Eine weitere Einsatzmöglichkeit besteht bei Grossereignissen und Katastrophen zur Sicherstellung einer zeitgerechten Hilfeleistung in Ausnahmesituationen in Ergänzung zu den bestehenden Einsatz-Organisationen.

Eine wichtige Voraussetzung für die Erweiterung der Einsatzindikationen ist aber eine genügende und gezielt auf die verschiedenen, zum Teil sehr schwierigen Situationen ausgerichtete Ausbildung. Dies ist nicht nur ein Muss, sondern auch ein wichtiges Zeichen der Wertschätzung für die freiwillige, unentgeltliche und auf Menschlichkeit beruhende Einsatzbereitschaft der First Responder.

\section{Bildnachweis}

(c) First Responder BE

\section{Literatur}

1 Saner H, Morger C, Eser P, von Planta M. Dual dispatch early defibrillation in out-of-hospital cardiac arrest in a mixed urban-rural population. Resucitation. 2013;84(9):1197-2012.

2 Mauri R, Burkart R, Benvenuti C, Caputo ML, Moccetti T, Del Bufalo A, Gallino A, Casso C, Anselmi L, Cassina T, et al. Better management of out-of-hospital cardiac arrest increases survival rate and improves neurological outcome in the Swiss Canton Ticino. EP Europace. 2016;18(3):398-404.

3 Leitfaden zum Aufbau und Betrieb von First Responder-Systemen. Interverband für Rettungswesen 2018. www.ivr-ias.ch/dokumente

4 Liste First Responder Netzwerke (2019). Schweizerische Herzstiftung. www.swissheart.ch

\section{Das Wichtigste in Kürze}

- $\quad$ First Responder (FR) sind Freiwillige, die als Helfer bei Herznotfällen eingesetzt werden, wobei kein professionelles medizinisches Wissen vorausgesetzt wird. Bei ausgelöstem Alarm ist der Zeitfaktor für die Hilfeleistung von zentraler Bedeutung.

- Das Einsatzpotential von FR geht aber über Herznotfälle hinaus: Die Autoren schlagen vor, dass das Einsatzgebiet der Ersthelfer auf häusliche Notfälle sowie auf Grossereignisse und Katastrophen ausgeweitet wird.

- Bei häuslichen Notfällen sollen FR zum Einsatz kommen, wenn keine Kontaktperson oder professionelle Hilfe zeitnah verfügbar ist. Bei Grossereignissen und Katastrophen sollen sie zur Betreuung von leicht- oder unverletzten Personen zum Einsatz kommen oder wenn die personellen Ressourcen der offiziellen medizinischen Notfallorganisation ausgeschöpft sind.

\section{L'essentiel en bref}

- Les premiers répondants (PR) sont des volontaires déployés comme assistants en cas d'urgence cardiaque, aucune connaissance médicale professionnelle n'étant requise. Le facteur temps est d'une importance capitale lorsqu'il s'agit d'alerter les services d'urgence.

- Le potentiel de déploiement des PR va toutefois au-delà des urgences cardiaques: les auteurs proposent que les premiers répondants interviennent aussi en cas d'urgences domestiques, d'événements et catastrophes majeurs.

- II convient de faire appel aux PR pour les urgences domestiques si aucune personne de contact ou aide professionnelle n'est rapidement disponible. Lors d'événements et de catastrophes majeurs, les PR doivent être déployés pour prendre en charge les personnes légèrement ou non blessées ou lorsque les ressources en personnel des services officiels d'urgence sont épuisées. 